\title{
CRISPR-Cas9 Human Genome Editing: Challenges, Ethical Concerns and Implications
}

\section{Otieno MO*}

Hekima University College in Nairobi, Kenya

*Corresponding author: Otieno MO, Hekima University College in Nairobi, Kenya, Tel: +254707044392, E-mail: mikeotson@yahoo.com

Received date: December 10, 2015; Accepted date: December 23, 2015; Published date: December 26, 2015

Copyright: $@ 2015$ Otieno MO. This is an open-access article distributed under the terms of the Creative Commons Attribution License, which permits unrestricted use, distribution, and reproduction in any medium, provided the original author and source are credited.

\begin{abstract}
Genome editing technologies may in the future have therapeutic potential for various incurable diseases: cancer, genetic disorders, HIVIAIDS to mention the most obvious. Genome editing of somatic cells, which is at it various clinical stages, is a promising area of therapeutic development. This year, a group of Chinese researchers led by Junjiu Huang - a gene-function researcher at Sun Yat-sen University in Guangzhou, used complex enzyme-editing tool CRISPR-Cas9 as a therapeutic agent to eradicate the human $\beta$-globulin (HBB) gene from the germline of the human embryo. The mutations in HBB gene cause $\beta$-thalassaemia (a deadly blood disorder). The research was, however, not completely successful, and had to be abandoned at its preliminary stage. This research was published in the journal Protein and Cell after it was rejected by the journal Nature and Science on ethical grounds. Caution flags have been raised about the use of CRISPR-Cas9 on human germline editing. This research has generated the debate among the world-renowned scientists about the ethical concerns and implications of CRISPR-Cas9 human germline editing. While some members of the scientific community have argued that a moratorium should be called on human germline editing, others have argued that it is unethical to withhold a technology that would eliminate devastating genetic diseases. This paper critically evaluates the challenges, ethical concerns and implications of CRISPR-Cas9 human germ line editing.
\end{abstract}

Keywords: Human genome editing; CRISPR-Cas9

\section{Introduction}

CRISPR-Cas9 is a genome-editing tool that is used to target a particular deleterious and disease causing genes in certain genetic disorders [1]. The targeted genes are altered, which brings about the changes in the germline intended to be bequeathed to the next generation so that the disease causing genes can be completely eradicated [1]. Genome editing of somatic cells, which is at it various clinical stages, is a promising area of therapeutic development. Recently, a group of Chinese researchers led by Junjiu Huang - a genefunction researcher at Sun Yat-sen University in Guangzhou, used complex enzyme-editing tool CRISPR-Cas9 as a therapeutic agent to eradicate the human $\beta$-globulin (HBB) gene from the germ line of the human embryo. The mutations in HBB gene cause $\beta$-thalassaemia (a deadly blood disorder).

The research was, however, not completely successful. So, it had to be abandoned at its preliminary stage. Huang headed off the ethical concerns raised by the research. He assured the scientific community that they used "non-viable" embryos obtained from local fertility clinics in their research. The embryos could not result in a live birth because they were fertilized by two sperms giving rise to triponuclear zygotes [2]. This research was published in the journal Protein and Cell after it was rejected by the journal Nature and Science because of ethical considerations involved [2,3]. The genome editing technologies currently in various clinical development stages are limited to modification of genetic material of somatic cells [4]. Since the techniques of genome editing raise a possibility of unpredictable outcomes, some scientists have argued that cure by genetic engineering techniques should be limited to genome editing of somatic cells [4].
While CRISPR-Cas9 embryo genome editing could completely eradicating genetic diseases, scientists have warned that it should be treated with caution. George Daley, a stem-cell biologist at Harvard Medical School in Boston, Massachusetts, raised an alarm regarding this research. He argues that: "I believe this is the first report of CRISPR-Cas9 applied to human pre-implantation embryos and as such the study is a landmark, as well as a cautionary tale. Their study should be a stern warning to any practitioner who thinks the technology is ready for testing to eradicate disease genes" [3]. Since germline modification causes genetic changes to the embryos, changes that are heritable, this technique can have unpredictable effects to the future generations. Moreover, unethical uses of the technique could emerge from gene editing of the human embryos [3]. This paper critically evaluates the challenges, ethical concerns and implications of CRISPRCas9 human germline editing.

\section{The biology and therapeutic potential of CRISPR-Cas9}

CRISPR-Cas9 found its origin from type II CRISPR-Cas systems, which enable the bacteria to mount an adaptive immunity against invading viruses and plasmids. When viruses or plasmids enter into a bacterial cell, CRISPR system allows the integration of the short viral DNA molecules into the CRISPR locus. The biogenesis of CRISPR RNA involves the transcription of CRISPR sequence into RNA, which is subsequently used with proteins encoded by Cas genes to form interference complexes that are used in the formation of RNA molecules to base pair with matching sequences of viral DNA. The CRISPR sequence CRISPRs, "clustered regularly interspaced short palindromic repeats", are short DNA repeats of viral origin found in the bacterial genome. Cas (CRISPR-associated) is an endonuclease that recognizes and cut the DNA [5]. CRISPR-Cas complex recognizes the 
DNA of the invading virus and guides the Cas protein to cleave the virus.

In 2013, Feng Zhang opened the window through which genome editing became a therapeutic possibility [6] when he engineered a novel version of CRISPR-Cas9 to edit human genomes [7]. The speed and efficiency of CRISPR-Cas9 is a remarkable leap in research. This feature can enable it to increase the identification of genes that are associated with human diseases and facilitate the development of therapies to correct the mutated gene [8]. Due to its unparalleled genetic specificity, scientists are using CRISPR-Cas9 genomic editing technology to facilitate discoveries in cancer biology. Cancer models have been developed using CRISPR-Cas9. The models better reflect the disease in humans [6]. Feng Zhang and Nobel laureate Phillip Sharp successfully engineered a mice model using CRISPR-Cas9 to model the deleterious effects of mutations in cancer. The ability of their system to introduce loss of function mutations in tumor suppressor genes and gain of function in proto-oncogenes facilitate screening of causal genetic mutations [6].

\section{Challenges, concerns and ethical implications of CRISPR- Cas9 Human genome editing}

While some members of the scientific community have argued that a moratorium should be called on human genome editing [9], others have argued that it is unethical to withhold a technology that would eliminate devastating genetic diseases, such as cystic fibrosis [10]. The Chinese researchers who used CRISPR-Cas9 genome editing technology to eradicate the human $\beta$-globulin (HBB) gene from the germline of the human embryo were confronted with some challenges, which made them to stop the research prematurely.

They discovered off-target mutations in the genome. Off-target mutations are unintended mutations in the genome. They occur when CRISPR-Cas9 cleaves other DNA sequences within the genome that are homologous to the target DNA sequences. These mutations can be deleterious. Off-target mutations can cause cell death or transformation [11]. The researchers found out that the mutations from CRISPR-Cas9 gene editing of the embryo were much higher than those observed in the mouse or human adult cells [3]. Huang and his team observed unintended mutations only in the exome of the genome. So, Huang argues that they would have got many more mutations had they done the whole genome sequence [2]. Due to some of the limitation of the research, Edward Lanphier said that the research should be stopped to allow a broad based discussion about the direction of where we are going [3]. Nevertheless, off-target mutations can be lessened or avoided by using the most recent CRISPR-Cas9 developed by Yang L, et al [12]. They increased the CRISPR-Cas9 efficiency in site-specific gene targeting using Cas9-modified hiPSC clones.

The cost of germline editing technology is very high to the extent that families coming from rich countries could afford it. The developing countries will not be in a position to afford the cost of this technology. This may confer an advantage to children born in developed countries.

Genome editing in human embryos using CRISPR-Cas9 could have unpredictable effects to the future generations. CRISPR-Cas9 technology could be used for non-therapeutic modifications [4]. This procedure will open the door to the loss of human diversity and eugenics [13]. Last year, Yoshimi et al., successfully changed the coat colour of the rat suggesting the possibility of inducing a pigmentation change in humans through embryonic editing [14]. So, the genetic enhancement of a specific appearance could cause substantial physical and mental health to the children since their appearance is imposed on them through means other than blood relationship [14].

Genome editing of the human embryo could hinder the ongoing research that involve gene editing of somatic cells that hold promise for therapeutic development. As rightly pointed out by Edward Lanphier et al [4], the public outcry about the ethical breach of human embryo genome editing could hinder the promising area of therapeutic development that are involved in making genetic changes in somatic cells. And there should be an open discussion around the appropriate action should a compelling case arise for therapeutic benefit of germline modification [4].

The nuclease may not be as efficient. The nuclease may not necessarily cleave both copies of the target gene or the cells may start dividing before the corrections are completed, resulting in genetic mosaic [4]. Mosaicism is the presence of the populations of somatic cells that are genetically distinct in an organism. Mosaicism is frequently masked. However, mosaicism can cause major phenotypic changes and reveal the expression of lethal genetic mutations [15]. Some of the genetic disorders that result from mosaicism include: Down syndrome, Klinefelter syndrome and Turner syndrome.

CRISPR-Cas9 genome editing technology to an embryo is a very risky affair. Researchers may not be in a position to determine, with precision, the effect of such procedures before birth. Lanphier opines that the quality control can be performed only on a subset of cells. This limitation shows that it may be impossible to know the effect of genetic modification of an embryo with precision until after birth. Even then, potential problems may take years to surface [4].

Another question that may arise regarding the embryo genome editing using CRISPR-Cas9 editing technology is the fate of the child produced by such technologies? While it is clear that people's informed consent is secured before genetically engineered somatic cells are used in clinical research, it is not clear what information would be needed from the prospective parents to adequately inform them about the risks involved in germline modification [4].

The scientific community should engage in a dialogue to establish guidelines of research involving genetic modification of human germ cells. The discussions should involve stakeholders in different fields: the general public, scientists, bioethicists, public policy and legal experts. The discussion should make a clear distinction between genome editing in germ cells and in somatic cells. The significant progress being made in clinical development of approaches to cure deleterious diseases should not be impeded by concerns regarding the ethical implications of germline editing [3].

A voluntary moratorium should be called on genetic modification of human germ cells. The US National Institute of Health has taken the lead in calling moratorium on genome editing of human embryos. Earlier this year, the director of US National Institutes of Health, Francis Collins issued a statement that banned NIH-funded research into genomic editing of human embryos [16]. Other countries should follow suit. A moratorium has been the kernel of debate among the members of the scientific community.

Some scientists argue that there is no need for a moratorium. John Harris, a bioethicist at the University of Manchester, UK is in support of the Huang's research on genetic modification of human germ cells using CRISPR-Cas9 genome editing technology. Since Huang and his 
team of researchers avoided ethical concerns that their research could have generated by using non-viable embryos that could not have led to a live birth, John Harris applauds the research arguing that it is no worse than what happens frequently in IVF where non-viable embryos are discarded. Therefore, he does not see the need for justification on moratorium [17]. Supporting the CRISPR-Cas9 human germline editing, Linzhao Cheng, a professor at Johns Hopkins University, argues: "Many people are concerned that we shouldn't be doing this, even in abnormal embryos that would be arrested at the blastocyst stage [as was the case here] and otherwise would be discarded. If many people have deep concerns about doing it even in non-viable embryos then how will we ever find out whether using a normal embryo would be better or worse?" [18].

Other scientists have argued that modification of germline cells could be justifiable if its purpose is solely for research. George Daley, a stem-cell biologist at Harvard Medical School in Boston, Massachusetts, succinctly points out that scientific research done using CRISPR-Cas9 and other genome editing tools in human germline cells could provide answers to many scientific questions, which are not related to clinical applications [17].

According to Harris, the side effects of germline editing should not be used as a justification to call a moratorium on genetic modification of human germ cells. It may be ethically justifiable to make the technique available in clinics. He argues that the genetic disease may be worse than the side effects because people with genetic disease will go on reproducing [17] and their progeny stand a higher chance of inheriting the defective gene responsible for a genetic disorder.

\section{Conclusion}

While CRISPR-Cas9 genome editing technology holds promise to personalized medicine, human genetic modification and the development of new drugs, the technology has raised caution flags. Genome editing technology is a cautionary tale. We can easily get caught up in the glamour of scientific and technological advancement while at the same time oblivious to the ethical ramification of such scientific and technological advancement. Some scientists have expressed concern that human germline editing has not only crossed the ethical redline; it is also fraught with many challenges. The recent research by Chinese scientists using CRISPR-Cas9 to edit the embryo genome was not completely successful. So, it had to be abandoned at its preliminary stage. There were off-target mutations in the genome. These off-target mutations can be deleterious as they can cause cell death and transformation. Consequently, embryo germline editing could be exploited in non-therapeutic research. For instance, it can be used to produce designer babies by eliminating undesired qualities and replacing them with desired ones. However, genome editing technology should not hinder the promising area of therapeutic development that are involved in making genetic changes in somatic cells. Due to the challenges and ethical concerns raised by CRISPRCas9 genome editing technology, a temporary moratorium should be called on the technology to allow the scientific community and other stakeholders to engage in a broad-based discussion to map the way forward for this technology.

\section{References}

1. Krishan K, Kanchan $\mathrm{T}$ (2015) Human genome editing and ethical considerations. Sci Eng Ethics.

2. Liang P, Xu Y, Zhang X, Ding C, Huang R, et al. (2015) CRISPR/Cas9mediated gene editing in human tripronuclear zygotes. Protein and Cell 6: 363-372.

3. Cyranoski D, Reardon S (2015) Chinese scientists genetically modify human embryos Nature News.

4. Lanphier E, Urnov F, Haecker SE, Werner M, Smolenski J (2015) Don't edit the human germ line. Nature 519: 410-411.

5. Doudna J, Charpentier E (2014) The new frontier of genome engineering with CRISPR-Cas9. Science 346: 1258096.

6. Chin A (2015) CRISPR-Cas9 Therapeutics: A Technology Overview. Oxford Biotechnolgy.

7. Cong L, Ran FA, Cox D, Lin S, Barretto R, et al. (2013) Multiplex Genome Engineering Using CRISPR/Cas Systems. Science 339: 819-823.

8. Qi LS, Larson MH, Gilbert LA, Doudna JA, Weissman JS (2013) "Repurposing CRISPR as an RNA- Guided Platform for SequenceSpecific Control of Gene Expression." Cell 152: 1173-1183.

9. Wade N (2015) Scientists seek ban on Method of Editing the Human Genome. The New York Times.

10. Gallagher J (2015) Embryo engineering a moral duty, says top scientist. BBC News.

11. ZhangF, Wen, Guo X (2014) CRISPR/Cas9 for genome editing: progress, implications and challenges. Hum Mol Genet 23: R40-46.

12. Yang L, Dennis G, Wang G, JohnA, Cheng-Zhong Z, et al. (2014) Targeted and genome-wide sequencing reveal single nucleotide variations impacting specificity of Cas9 in human stem cells. Nature Commun 5: 1-6.

13. Yang A (2015) Thinking towards the future of CRISPR/Cas9. Brevia.

14. Ishi $\mathrm{T}$ (2015) Germ line genome editing in clinics: the approaches, objectives and global society. Brief Funct Genomics: pii: elv053.

15. Youssoufian H, Pyeritz RE (2002) Mechanisms and consequences of somatic mosaicism in humans. Nature Reviews Genetics 3: 748-758.

16. Collins F (2015) Statement on NIH funding of research using geneediting technologies in human embryos. The NIH Director - National Institutes of Health (NIH).

17. Sarah R (2015) Ethics of embryo editing paper divides scientists, Nature News.

18. Entine Jon (2015) Ethical and regulatory reflections on CRISPR gene editing revolution. Genetic Literacy Project. 